\title{
正向电压对氢化锆微弧氧化阻氢膜层性能的影响
}

\author{
张鹏飞 ${ }^{1,2}$, 间淑芳 ${ }^{1}$, 陈伟东 ${ }^{1}$ ，李世江 ${ }^{1}$, 耿艳花 ${ }^{1}$ ，王宏兴 ${ }^{2}$
}

(1. 内蒙古工业大学 材料科学与工程学院, 呼和浩特 $010051 ; 2$. 西安交通大学 宽禁带半导体材料与器件研究中 心, 西安 710049)

摘 要: 在偏铝酸钠-氢氧化钠-EDTA 混合溶液中, 采用恒压模式对氢化锆表面进行微弧氧化处理。借助场发射扫 描电镜(FE-SEM)、X 射线衍射仪(XRD)及真空脱氢实验, 研究了不同正向电压 $(350 \sim 425 \mathrm{~V})$ 对微弧氧化膜层表面、 截面、相结构及阻氢渗透性能的影响。分析结果表明: 微弧氧化过程大致由阳极氧化、火花放电、微弧氧化和熄弧 四个阶段组成。制备的膜层分为致密层和疏松层, 致密层所占比例约为 $80 \%$ 。随着正向电压的增大, 晶粒尺寸增大, 晶面间距减小, 氢化锆表面微弧氧化膜层厚度由 $122 \mu \mathrm{m}$ 增加至 $150 \mu \mathrm{m}$, 膜层的增长速度也随正向电压的增大而加 快。但正向电压的改变对膜层的相结构并无显著影响, 膜层由单斜相氧化锆和四方相氧化锆组成, 当正向氧化电压 为 $400 \mathrm{~V}$ 时, 氧化膜的 PRF 值达到最大值 20 。

关 键 词: 氢化锆; 微弧氧化; 正向电压; 阻氢渗透层

中图分类号: TQ174 文献标识码: A

\section{Anode Voltage on Hydrogen Permeation Barrier Performance of Zirconium Hydride with Micro-arc Oxidation}

\author{
ZHANG Peng-Fei ${ }^{1,2}$, YAN Shu-Fang ${ }^{1}$, CHEN Wei-Dong ${ }^{1}$, \\ LI Shi-Jiang ${ }^{1}$, GENG Yan-Hua ${ }^{1}$, WANG Hong-Xing ${ }^{2}$
}

(1. College of Materials Science and Engineering, Inner Mongolia University of Technology, Hohhot 010051, China; 2. Institute of Wide Band Gap Semiconductors, Xi'an Jiaotong University, Xi'an 710049, China)

Abstract: Technology of micro-arc oxidation was applied on $\mathrm{ZrH}_{1.8}$ in the electrolyte composed of $\mathrm{NaAlO}_{2}+\mathrm{NaOH}^{+}$ $\mathrm{Na}_{2}$ EDTA in constant voltage mode. The film was characterized by field emission scanning electron microscope (FE-SEM), X-ray diffraction (XRD), and vacuum dehydrogenation experiment, respectively. The influences of anode voltage (350 to $425 \mathrm{~V}$ ) on surface and cross-sectional morphology, structure, and hydrogen resistance properties of the hydrogen resistance film on the surface of $\mathrm{ZrH}_{1.8}$ were investigated. The micro-arc oxidation process can be divided into four stages: anodic oxidation, spark discharge, micro-arc oxidation and flameout. The results reveal that film obtained in the electrolyte is composed of inner dense layer and outer loose layer, and proportion of the dense layer is about $80 \%$. Grain sizes and growth rate of the film increase while the d-spacing decreases with the increase of the anode voltage, and the thickness of the film is increased from 122 to $150 \mu \mathrm{m}$. However, anode voltage has no obvious influence on the structure of the film. The prepared film is composed of $\mathrm{M}-\mathrm{ZrO}_{2}$ and $\mathrm{T}-\mathrm{ZrO}_{2}$. When the anode voltage is $400 \mathrm{~V}$, the permeation reduction factor value reaches the maximum of 20.

Key words: zirconium hydride; micro-arc oxidation; anode voltage; hydrogen permeation barrier

收稿日期: 2017-07-05; 收到修改稿日期：2017-09-06

基金项目: 国家自然科学基金(51164023，51364026); 内蒙古自然科学基金(2016MS0505); 内蒙古工业大学科学研究项目 (X201410)

National Natural Science Foundation of China (51164023, 51364026); Natural Science Foundation of Inner Mongolia Autonomous Region (2016MS0505); Science Project of Inner Mongolia University of Technology (X201410)

作者简介: 张鹏飞(1992-), 男, 硕士研究生. E-mail: pfzhang.kk@foxmail.com

通讯作者: 间淑芳, 博士. E-mail: ysfch@163.com 
高原子比 $(\mathrm{H} / \mathrm{Zr})$ 的氢化锆因具有低中子吸收截 面、高热导率及负温度反应性系数, 能够保持良好 的固有安全性能, 成为空间反应堆理想的固体慢化 剂及屏蔽材料 ${ }^{[1-4]}$ 。但由于氢化锆存在分解压, 当工 作温度超过 $873 \mathrm{~K}$ 时, 氢平衡压会超过 $10 \mathrm{kPa}$, 进 而造成氢的大量析出, 致使氢化锆作为慢化剂的工 作寿命缩短。因此, 如何解决氢化锆在工作温度下 的氢损失问题, 延长氢化锆慢化剂的服役寿命, 成 为亟待解决的问题 ${ }^{[5-6]}$ 。

为了顺利实现氢化锆慢化剂在反应堆中的应用, 国内外普遍采用表面技术制备氧化物、碳(氮)化物 或金属阻氢渗透膜层 ${ }^{[7-9]}$ 等来控制氢化锆慢化剂的 失氢行为。微弧氧化(Micro-Arc Oxidation, MAO)表 面处理技术是由阳极氧化技术发展而来, 由于具有 流程简单, 成膜效率高和制备膜层的机械性能良好 等特点，广泛应用于 $\mathrm{Mg} 、 \mathrm{Al}$ 等阀金属表面得到氧 化物保护性涂层 ${ }^{[10-14]}$ 。一般通过控制正负向电压、 氧化时长、电解液成分、占空比等参数调节成膜质 量。研究表明, 增加正向电压对 MAO 膜层的成膜 厚度、形貌和抗腐蚀性等有不同的影响规律 ${ }^{[15-16]}$ 。 在锆基合金中使用磷酸盐及硅酸盐体系制备 MAO 膜层已有相关报道，但存在生长速率低、孔隙率高等 缺点 ${ }^{[17-18]}$ 。而铝酸盐电解液体系不仅能够改善膜层 致密性, 且对环境不会造成污染 ${ }^{[19-20]}$ 。因此, 本实验 在铝酸盐体系下进行，通过对不同正向电压下制备 的氢化锆 MAO 膜层结构及阻氢渗透效率进行表征, 以期对研究铝酸盐体系下氢化锆 MAO 成膜的质量 及阻氢性能提供指导意义。

\section{1 实验方法}

\section{1 实验材料}

实验准备阶段, 使用数控线切割机将圆柱形氢 化锆基体(北京有色金属研究总院提供)线切割成尺 寸为 $\phi 20 \mathrm{~mm} \times 2 \mathrm{~mm}$ 的试样, 为减少尖端放电现象, 对试样尖锐处进行倒角/圆滑处理后, 再经过砂纸抛 光至 1000 号。使用高频超声波清洗器, 将试样分别 在丙酮、酒精、去离子水中进行超声清洗 $15 \mathrm{~min}$, 除 去试样表面油脂后烘干待用。

\section{2 实验方法}

实验使用 WHD-30 型双极性交流脉冲微弧氧化 装置, 采用去离子水配置偏铝酸钠电解液体系, 将 不锈钢槽壁作为阴极, 氢化锆试样作为阳极进行微 弧氧化实验。通过循环水冷温度控制系统将电解液 温度保持在 $25^{\circ} \mathrm{C}$ 以下，正向电流、负向电流随反应 进行自动调节, 具体实验参数如表 1 所示。
表 1 微弧氧化实验参数

Table 1 Experimental parameters of micro-arc oxidation

\begin{tabular}{ccccc}
\hline No. & Time/min & $\begin{array}{c}\text { Anode } \\
\text { voltage/V }\end{array}$ & $\begin{array}{c}\text { Cathode } \\
\text { voltage/V }\end{array}$ & $\begin{array}{c}\text { Electrolyte } \\
\text { composition }\end{array}$ \\
\hline 1 & & 350 & & \\
2 & \multirow{2}{*}{10} & 375 & 120 & $\mathrm{NaAlO}_{2}$ \\
3 & & 400 & & $\mathrm{NaOH}$ \\
4 & & 425 & & $\mathrm{Na}_{2} \mathrm{EDTA}$ \\
\hline
\end{tabular}

采用场发射扫描电子显微镜(FE-SEM, FEI Quanta FEG 250) 对试样表面形貌进行观察; 采用场发射 扫描电子显微镜(FE-SEM, HITACHI SU-8010) 对 试样截面形貌进行观察; 通过 X 射线全自动粉末衍 射仪(XRD, 荷兰 $x$ 'Pert Pro MRD) 对氢化锆 MAO

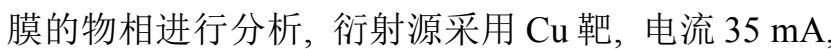
电压 $40 \mathrm{kV}$ ，扫描范围 $2 \theta=20^{\circ} \sim 80^{\circ}$, 步进扫描 $0.02^{\circ}$ 。 对试样进行真空脱氢实验, 测试氢化锆表面 MAO 膜层的阻氢渗透效率, 实验将需要评价阻氢性能的 氢化锆试样放置在真空度为 $10^{-4} \mathrm{~Pa}$, 温度为 $650^{\circ} \mathrm{C}$ 的环境下保温 $40 \mathrm{~h}$, 然后通过测量试样的失氢量来 评价 MAO 膜层的阻氢渗透效率。氢化锆表面 MAO 膜层的阻氢渗透效果用氢渗透降低因子 PRF 值 ${ }^{[21]}$ (Permeation Reduction Factor)表征。

\section{2 结果与讨论}

\section{1 恒压模式下铝酸盐体系氢化锆 MAO 过程}

在恒压模式下，正向电压固定为 $400 \mathrm{~V}$, 负向电 压固定为 $120 \mathrm{~V}$, 频率为 $200 \mathrm{~Hz}$ 的反应情况通过分 析电流随反应自动调节的变化情况, 研究膜层的生 长过程。图 1 为正向电流随时间变化的曲线, 如图 所示, 正向峰值电流为 $7 \mathrm{~A}$, 电流整体呈现先增加 后减少的变化趋势, 并在 $450 \mathrm{~s}$ 后趋于平稳。 $\mathrm{MAO}$ 过程总体可分为四个阶段: (1)普通阳极氧化,即正向 电流以超过 $7 \mathrm{~A} / \mathrm{min}$ 的速度迅速升至峰值电流，如 图 1 中 I 阶段所示, 此阶段材料表面覆盖大量气泡, 氢化锆试样失去金属光泽; (2)火花放电, 即阶段 II 经历正向电流下降的过程, 氢化锆试样表面随机出 现游动的白色细淢火花; (3)微弧氧化, 如阶段III所 示, 正向电流下降速度略有加快, 表面产生耀眼的 金黄色弧斑; (4)熄弧阶段，在阶段 IV 即 $450 \mathrm{~s}$ 以后, 电流进入平稳阶段, 弧斑减弱至消失, 但仍有部分 细小火花, 说明微等离子体现象依然存在。

普通阳极氧化阶段主要为表面氧化, 在高电压 高场强作用下，氢化锆表面首先形成氧化锆绝缘薄 膜, 电流迅速上升, 表面产生的大量气泡为发生等 


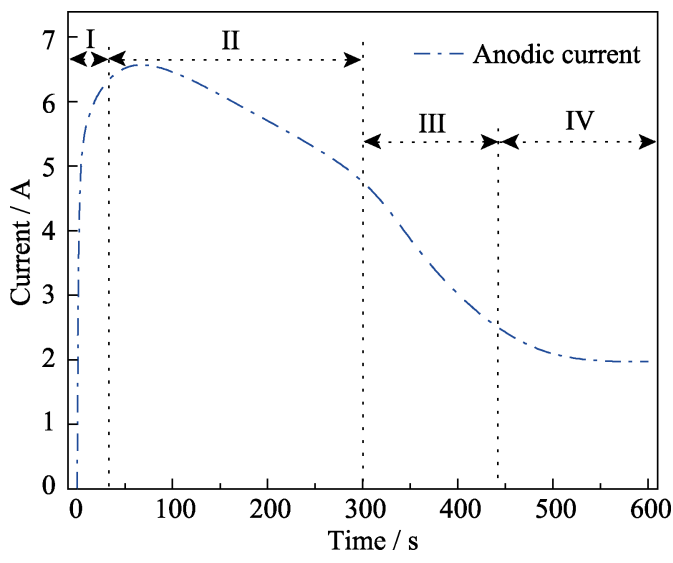

图 1 铝酸盐体系下 $\mathrm{MAO}$ 过程中正向电流随反应时间变化 的曲线

Fig. 1 Anodic current-time responses during MAO process in $\mathrm{NaAlO}_{2}$ electrolyte

离子体提供了条件。一旦超过临界击穿电压, 微弧 氧化便进入火花放电过程, 初生氧化锆绝缘薄膜将 被电击穿, 并产生游动的白色火花, 微区伴随着瞬 间的高温高压熔融了氧化膜, 又在电解液的急冷作 用下迅速凝固形成孔洞。击穿会偏向于在氧化膜较 薄位置进行，这是表面弧光表现出随机游动的原 因。随着膜层增厚，电流不断下降，反应进入微弧氧 化阶段。膜层孔洞互相连接或自我扩大，形成物质 输送和能量传递的反应通道，使反应不断向深层进 行。膜厚的持续增加使膜层击穿变得困难, 电流变 化缓慢，此时反应进入熄弧阶段。

\section{2 正向电压对氢化锆 MAO 膜层物相的影响}

图 2 为使用偏铝酸钠作为主成分的电解液体系, 通过改变不同正向电压进行微弧氧化过程, 制备得 到的阻氢渗透膜层的 XRD 图谱。分析表明, 正向电 压的增大对膜层的物相影响不大, 膜层均主要由单 斜相氧化锆 $\left(\mathrm{M}-\mathrm{ZrO}_{2}\right)$ 和四方相氧化锆 $\left(\mathrm{T}-\mathrm{ZrO}_{2}\right)$ 组成, 通过 JADE6.5 进行定量分析发现, 四方相氧化锆占 比会随着正向电压的升高而增加，当电压由 $350 \mathrm{~V}$ 增大至 $425 \mathrm{~V}$ 时, 其占比由 $38.1 \%$ 增加至 $46.3 \%$, 而 单斜相氧化锆占比由 $61.9 \%$ 下降至 $53.7 \%$ 。这是因 为正向电压的升高会导致反应发生时的热量增加, 作为高温相的四方相氧化锆含量增加, 并在随后的 冷却相变中更多的保留下来。两种氧化锆物相都具 有致密的组成结构, 对于阻氢渗透起到一定作用。 晶粒大小对材料的机械性能有一定影响, 大的晶粒尺 寸使材料拥有良好的可塑性，而纳米级别的晶粒则让 材料具有可靠的强度和其它良好的机械性能 ${ }^{[22]}$ 。对不 同正向电压下膜层中衍射峰最强峰(101)进行定量 分析，结果如表 2 所示，随着正向电压的增大，晶粒 尺寸变大, 晶面间距呈减小趋势, 表明结晶度随着

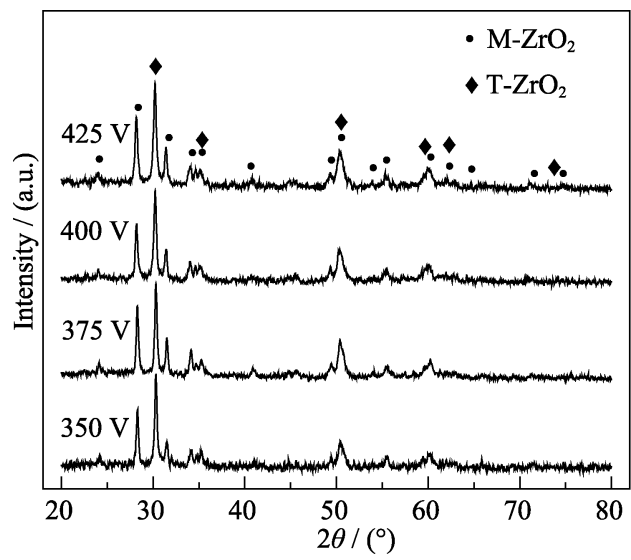

图 2 不同正向电压制备得到的氢化钻 $\mathrm{MAO}$ 膜层的 XRD 图谱

Fig. 2 XRD patterns of the MAO coatings on the surface of $\mathrm{ZrH}_{1.8}$ prepared at different anode voltages

表 2 不同正向电压下氢化锆 MAO 膜层的 晶粒尺寸和晶面间距

Table 2 Grain size and $d$-spacing of MAO coatings on the surface of $\mathrm{ZrH}_{1.8}$ prepared at different anode voltages

\begin{tabular}{ccccc}
\hline Voltage $/ \mathrm{V}$ & $2 \theta /\left(^{\circ}\right)$ & $d$-spacing $/ \mathrm{nm}$ & $\mathrm{FWHM} /\left(^{\circ}\right)$ & Grain size $/ \mathrm{nm}$ \\
\hline 350 & 30.194 & 0.29575 & 0.314 & 27.7 \\
375 & 30.214 & 0.29556 & 0.293 & 29.9 \\
400 & 30.293 & 0.29480 & 0.286 & 30.8 \\
425 & 30.332 & 0.29443 & 0.262 & 34.4 \\
\hline
\end{tabular}

正向电压的增大而提高，所制备的晶粒在纳米级别， 具有一定保护性。

\section{3 正向电压对氢化锆 MAO 膜层表面的影响}

图 3 为不同正向电压下得到的氢化锆 $\mathrm{MAO}$ 膜
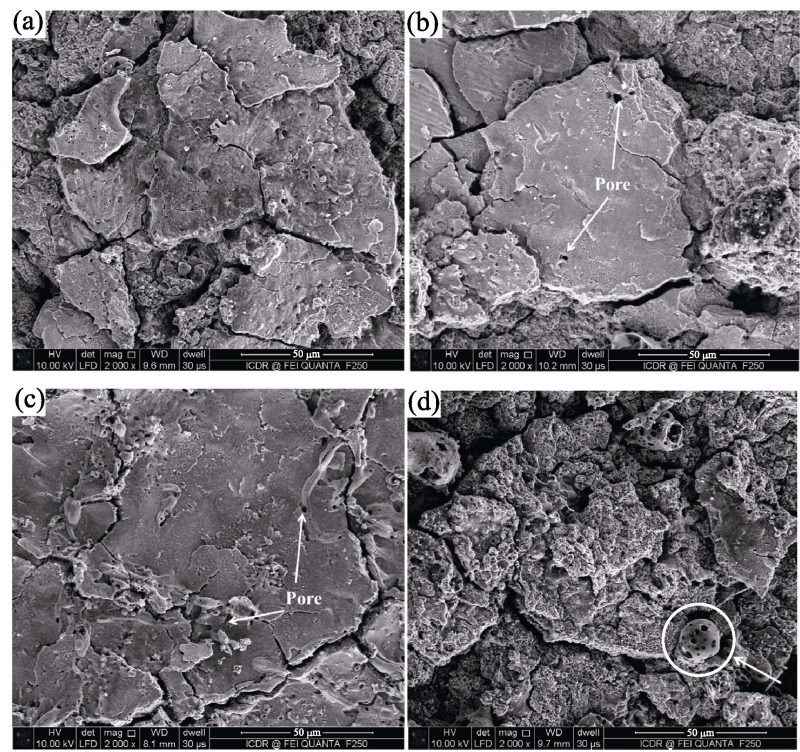

图 3 不同正向电压下制备的膜层的表面 SEM 照片

Fig. 3 Surface morphologies of oxide films prepared at different anode voltages

(a) $350 \mathrm{~V}$; (b) $375 \mathrm{~V}$; (c) $400 \mathrm{~V}$; (d) $425 \mathrm{~V}$ 
层的表面形貌照片, 如图所示, 在电压为 $350 \mathrm{~V}$ 时, 表面所呈现的微孔细小, 高温熔融物质流淌填补了 部分孔洞, 并在 “液淬” 作用下快速凝固, 从而形成 区域性平坦的表面。当正向电压升高至 $400 \mathrm{~V}$, 放电 微孔中电场驱动力与能量密度达到动态平衡, 熔融 氧化物不断填补微孔并凝固, 得到的膜层表面较为 平整。继续升高电压至 $425 \mathrm{~V}$, 高压使得击穿过程加 剧, 离子电流密度增加, 放电能量增强。放电反应产 生的高能等离子体击穿已经形成的膜层, 且更高的 热量和更多的熔融氧化物使得凝结困难, 难以覆盖 放电气孔，容易造成致密性下降，甚至出现图中“过 烧”现象。

\section{4 正向电压对氢化锆 MAO 膜层截面的影响}

使用 MAO 技术制备的氢化锆膜层截面如图 4 所示, 膜层由基体表面原位生长得到, 总体上可分 为致密层和疏松层, 其中致密层位于基体一侧, 孔 隙率较低, 以治金方式紧密结合; 疏松层位于膜层 外侧, 存在部分孔隙缺陷, 致密层与疏松层界限并 不清晰, 图 4(e)为图 4(a)膜层截面图的局部放大形 貌图。在普通阳极氧化阶段, 一些负离子如 $\mathrm{AlO}_{2}^{-}$和 $\mathrm{OH}^{-}$在电场作用下聚集在氢化锆基体表面。微弧氧 化技术会在基体材料上原位生长一层氧化膜 ${ }^{[23]}$ 。当 反应进入火花放电阶段, 氢化锆作为活泼电极优先 放电产生 $\mathrm{Zr}^{4+}$ 离子, 阴阳离子在以高电场强度为主 的多种驱动力作用下, 直接在基体表面发生氧化反 应生成氧化锆，因此膜层与氢化锆基体为治金结合 方式。随着正向电压的升高, 膜层平均厚度增加, 致 密层厚度也随之增加, 非化学计量式的氧化锆晶体
结构中氧、锆离子平均间隙小于氢原子的直径, 氢 难以通过致密完好的氧化膜, 因此较高的致密层厚 度有利于捕获氢向外扩散的行为, 从而有利于阻氢 析出 ${ }^{[24-25]}$ 。

\section{5 正向电压对氢化锆 MAO 膜层厚度及阻氢 性能的影响}

图 5 为不同正向电压下得到的膜层厚度、生长 速率及氢渗透降低因子变化关系曲线。从图 5 可以看 出, 膜层厚度伴随正向电压的升高, 由 $121.9 \mu \mathrm{m}$ 增加 到 $150.0 \mu \mathrm{m}$, 且在 $350 \sim 375 \mathrm{~V}$ 期间膜层厚度的生长 效率(膜层生长速率随时间变化的曲线斜率) 最高, 当正向电压超过 $375 \mathrm{~V}$ 时, 虽然膜层厚度继续增加, 但膜层生长效率下降。正向电压的增加使得氧化膜 两侧电场强度增大, 随之物质输送的驱动力增大, 电荷和产生的热量增加, 因而加快了电击穿过程和 氧化反应的发生过程。随着膜层的增厚, 击穿膜层 需要更大的电压支持, 且伴随着更多的热量产生, 导致膜层凝固效率降低, 从而降低膜层的生长效率。

不同正向电压制备的 MAO 膜层阻氢渗透性能 用氢渗透降低因子 PRF 值表征。如图 5 所示, 随着 正向电压的增加, 膜层的阻氢渗透能力呈现先增加 后减少的趋势, 当正向电压为 $400 \mathrm{~V}$ 时, PRF 值达到 最大值 20 , 继续增大正向电压, PRF 值有减少趋势。 分析认为, 随着膜层总体增厚, 致密层厚度增加, 膜层表面也趋于平整, 阻氢渗透能力增强。但是正 向电压的持续增大会导致击穿反应发生时电流破坏 能力的增强, 伴随着反应通道孔径增大, 反应生成 的熔融氧化物未能及时填充反应通道, 且高压造成

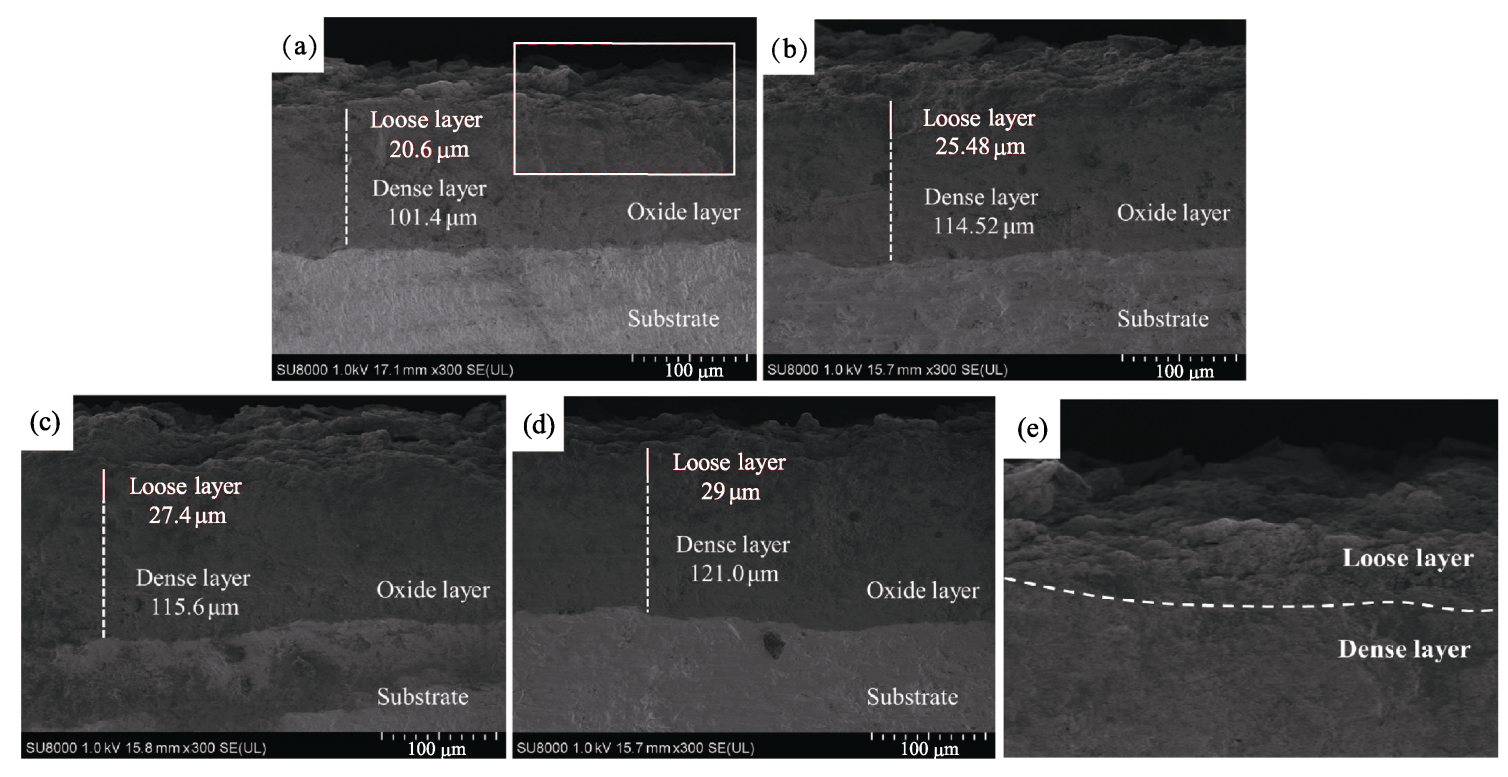

图 4 不同正向电压下制备得到的膜层的截面形貌

Fig. 4 Cross-section morphologies of oxide films prepared at different anode voltages (a) $350 \mathrm{~V}$; (b) $375 \mathrm{~V}$; (c) $400 \mathrm{~V}$; (d) $425 \mathrm{~V}$ and magnified image of selected area in (a) (e) 


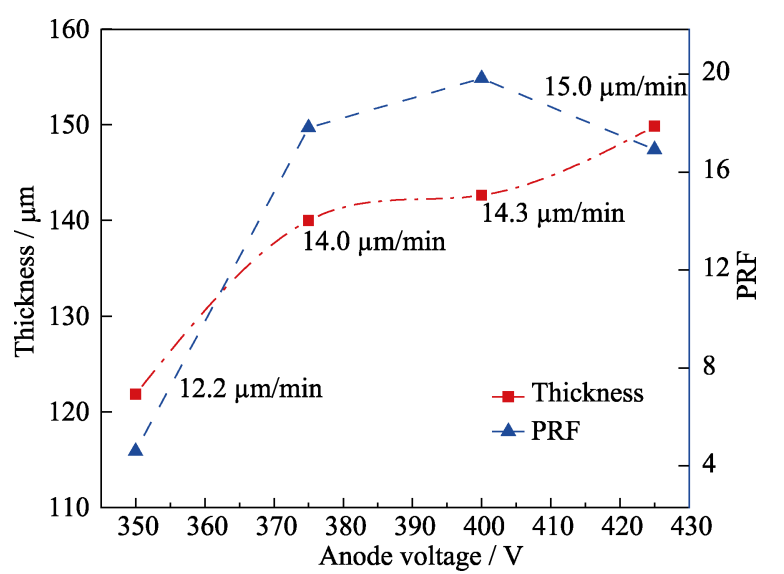

图 5 不同正向电压下制备得到的膜层厚度、生长速率及氢 渗透降低因子变化关系

Fig. 5 Thickness, growth rate and permeation reduction factor of oxide films prepared at different anode voltages

的高热量使生成物在电解液激冷作用下体积收缩不 均匀, 膜层表面及内部出现缺陷裂纹, 造成膜层致 密度降低, 进而阻氢渗透性能下降。

\section{3 结论}

1) 氢化锆在恒压模式下进行微弧氧化处理，正 向电流随反应时间增加有先增加后减小并趋于平稳 的趋势，反应过程大致可分为普通阳极氧化、火花放 电、微弧氧化及熄弧四个阶段。

2) 采用微弧氧化技术在铝酸盐体系中制备了氢 化锆表面阻氢渗透膜层, 膜层主要由单斜相氧化锆 和四方相氧化锆组成，随着正向电压增加，膜层表 面微孔增大，晶粒尺寸增大。

3) 氢化锆微弧氧化膜层由疏松层和致密层组成, 氧化膜外部为疏松层，靠近基体为致密层，膜层与 基体以冶金方式结合紧密。当正向氧化电压为 $400 \mathrm{~V}$ 时，氧化膜的 PRF 值达到最大值 20。

\section{参考文献:}

[1] OKA Y, JEVREMOVIC T, KOSHIZUKA S. Negative void reactivity in a large liquid-metal fast breeder reactor with hydrogenous moderator (ZrH1.7) layers. Nuclear Technology, 1994, 107(1): $15-22$.

[2] JEVREMOVIC T, OKA Y, KOSHIZUKA S. Effect of zirconiumhydride layers on reducing coolant void reactivity of steam cooled fast breeder reactors. Journal of Nuclear Science and Technology, 1993, 30(6): 497-504.

[3] ALLEN G B, KERR M, DAYMOND M R. Measurement and modeling of strain fields in zirconium hydrides precipitated at a stress concentration. Journal of Nuclear Materials, 2012, 430(1/2/3): 27-36.

[4] HAYASHI T, TOBITA K, NAKAMORI Y, et al. Advanced neutron shielding material using zirconium borohydride and zirconium hydride. Journal of Nuclear Materials, 2009, 386(2): 386-388.
[5] OLANDER D, GREENSPAN E, GARKISCH H D, et al. Uraniumzirconium hydride fuel properties. Nuclear Engineering and Design, 2009, 239(8): 1406-1424.

[6] KUMAR NAPK, SZPUNAR J A, HE Z. Preferential precipitation of hydrides in textured zircaloy-4 sheets. Journal of Nuclear Materials, 2010, 403(1/2/3): 101-107.

[7] OLANDER D. Nuclear fuels - present and future. Journal of Nuclear Materials, 2009, 389(1): 1-22.

[8] LIU X Z, HUANG Q R, DU J J, et al. Study on permeation of hydrogen through HR-1 austenitic stainless steel with $\mathrm{Cr}_{2} \mathrm{O}_{3}$ and TiN thin film coatings. Chinese Journal of Nuclear Science and Engineering, 1997, 17(3): 281-284.

[9] MA H R, FU X G, QIN B. Research on prevention method of hydrogen release of zirconium hydride at high temperature. Atomic Energy Science and Technology, 2016, 50(11): 2023-2026.

[10] CHEN Y L, XUE Z G, WANG Q, et al. New findings on properties of plasma electrolytic oxidation coatings from study of an Al-Cu-Li alloy. Electrochimica Acta, 2013, 107(3): 358-378.

[11] CHEN M A, OU Y C, YU C Y, et al. Corrosion performance of epoxy/BTESPT/MAO coating on AZ31 alloy. Surface Engineering, 2016, 32(1): 38-46.

[12] XIONG W M, NING C Y, GU Y H, et al. Effect of positive voltage on phase structure of micro-arc oxidation films of magnesium alloy. Rare Metal Materials and Engineering, 2011, 40(12): 2236-2240.

[13] KHANMOHAMMADI H, ALLAHKARAM S R, TOWHIDI N, et al. Preparation of PEO coating on $\mathrm{Ti}_{6} \mathrm{Al}_{4} \mathrm{~V}$ in different electrolytes and evaluation of its properties. Surface Engineering, 2016, 32(6): 448-456.

[14] MARTIN J, LEONE P, NOMINE A, et al. Influence of electrolyte ageing on the plasma electrolytic oxidation of aluminium. Surface and Coatings Technology, 2015, 269(1): 36-46.

[15] CHEN M, MA Y Z, MA Y, et al. Effects of voltage increment on performances of micro-arc oxidation coatings of magnesium alloys. Rare Metal Materials and Engineering, 2010, 39(11): 1943-1947.

[16] ABBASI S, BAYATI M R, GOLESTANI F F, et al. Micro arc oxidized $\mathrm{HAp}-\mathrm{TiO}_{2}$ nanostructured hybrid layers-part I: effect of voltage and growth time. Applied Surface Science, 2011, 257(14): 5944-5949.

[17] WANG S, GUO F, LIU L, et al. Effect of process parameters on thickness and morphology of micro-arc oxidation ceramic coating of zirconium alloy. Rare Metal Materials and Engineering, 2008, 37(8): 1466-1470.

[18] XUE W B, JIN Q, ZHU Q Z, et al. Preparation and properties of ceramic coating formed by microarc oxidation on zirconium alloy. Transactions of Materials and Heat Treatment, 2010, 31(2): 119-122.

[19] 吴汉华. 铝、钛合金微弧氧化陶瓷膜的制备表征及其特性研究. 长春: 吉林大学博士学位论文, 2004.

[20] FANG D R, WANG J H, YANG J, et al. Electrolyte optimization of microarc oxidation of magnesium alloy. Transactions of Materials and Heat Treatment, 2004, 25(5b): 1072-1075.

[21] ZHONG X K, YAN G Q, CHEN W D, et al. Effect of $\mathrm{Na}_{5} \mathrm{P}_{3} \mathrm{O}_{10}$ addition on the MAO coating on the surface of $\mathrm{ZrH}_{1.8}$. Rare Metal Materials and Engineering, 2012, 41(S2): 541-544.

[22] HE J, YE J, LAVERNIA E J, et al. Quantitative analysis of grain size in bimodal powders by X-ray diffraction and transmission electron microscopy. Journal of Materials Science, 2004, 39(23): 6957-6964.

[23] YANG G L, LV X Y, BAI Y Z, et al. The effects of current density on the phase composition and microstructure properties of microarc oxidation coating. Journal of Alloys and Compounds, 2002, 345(1/2): 196-200.

[24] 王俐艳. $\mathrm{CO}_{2}$ 反应法制备氢化锆表面氢渗透阻挡层的研究. 成 都: 西华大学博士学位论文, 2007.

[25] YAN S F, LIU X D, CHEN W D, et al. Influence of oxidation time on MAO film on the surface of $\mathrm{ZrH}_{1.8}$ in a phosphate system. Rare Metal Materials and Engineering, 2014, 43(7): 1717-1721. 